Access to this work was provided by the University of Maryland, Baltimore County (UMBC)

ScholarWorks@UMBC digital repository on the Maryland Shared Open Access (MD-SOAR) platform.

Please provide feedback

Please support the ScholarWorks@UMBC repository by emailing scholarworks-group@umbc.edu and telling us what having access to this work means to you and why it's important to you. Thank you. 


\title{
Nonlinear Optical Effects in Epsilon-Near-Zero Plasmonic Waveguides and Metamaterials
}

\author{
Christos Argyropoulos $^{(1)}$, G. D’Aguanno ${ }^{(2)}$, Andrea Alù ${ }^{(1)}$ \\ (1) The University of Texas at Austin, Department of Electrical and Computer Engineering, Austin, Texas, 78712 \\ Tel: + 1-512-471-5922, Fax: + 1-512-471-6598, Email: alu@mail.utexas.edu \\ (2) AEgis Technologies, Nanogenesis Division, 410 Jan Davis Dr. Huntsville, Alabama, 35806
}

\begin{abstract}
Nonlinear effects may be strongly enhanced in arrays of plasmonic waveguides operated at cutoff, which behave as near-zero-permittivity metamaterials. Their large phase velocity combined with uniform field enhancement are ideal for second-harmonic-generation and soliton propagation.
\end{abstract}

(C)2013 Optical Society of America

OCIS codes: 160.3918 Metamaterials, 160.4670 Optical Materials, 260.3910 Metal Optics, 250.5403 Plasmonics.

Arrays of plasmonic waveguides operated near their cut-off frequency form an effective near-zero permittivity metamaterial. Ideally, infinite phase velocity and perfect tunneling of the electromagnetic energy is achieved exactly at this frequency point [1]. In addition, the electric field is squeezed and enhanced inside the nanochannels [2]. These features, combined with optical nonlinearities, may lead to efficient and robust novel nonlinear optical nanodevices [3]. For example, recently, it was shown that epsilon-near zero (ENZ) plasmonic waveguides can effectively enhance the Kerr third-order nonlinear susceptibility by several orders of magnitude [3]. The nonlinearity enhancement is spatially extended through the ENZ nanochannels, and highly nonlinear efficiencies may be achieved combined with lower power need.

In our talk, we will extend these recent theoretical findings towards giant second harmonic generation and efficient temporal soliton propagation, when different nonlinear media are loaded inside the nanochannels of ENZ plasmonic waveguides. In the presented results, we will use the Transmission-Line (TL) method to analyze the anomalous tunneling properties of ENZ ultranarrow cross-slit waveguides loaded with second-order $\chi^{(2)}$ nonlinear materials. Giant second harmonic generation and frequency mixing can be achieved based on the proposed plasmonic structure. These useful properties are mainly due to the novel phase matching capabilities and the uniform enhanced fields inside the ENZ nanochannels, which represent ideal conditions to achieve high second harmonic and, in general, frequency mixing conversion efficiencies for backward and forward signals with theoretically infinite coherence length. Several applications are envisioned based on the proposed concepts, such as efficient electromagnetic wave mixers and parametric amplifiers operating in a wide frequency range.

We will extend the ENZ concept to the interesting effect of self-phase modulation of propagating optical pulses, which is possible when we load the nanochannels of the plasmonic waveguide with Kerr $\chi^{(3)}$ optical nonlinearities. In this case, the pulses' dispersion can be potentially balanced and this interesting property can lead to ultraslow guided light pulses. As a result, the electromagnetic pulses can effectively propagate without dispersion and with unaffected shape for very long propagation lengths, a very desirable property, especially in optical high-speed communications. These types of ultraslow optical pulses are called temporal solitons. In this presentation, we will demonstrate that nonlinear ENZ nanochannels can also sustain efficient temporal soliton waves (slow-light) for fiber-optic communication frequencies, triggered by much lower input intensities compared to conventional optical fiber systems. Hence, the efficiency of optical data storage may be largely enhanced. Interesting applications will be presented in detail during the conference, such as data synchronizers and robust slow-light systems.

\section{References}

[1] M. Silveirinha, N. Engheta, "Tunneling of Electromagnetic Energy through Subwavelength Channels and Bends using $\varepsilon$-Near-Zero Materials,” Phys. Rev. Lett. 97, 157403 (2006).

[2] A. Alù, N. Engheta, "Light squeezing through arbitrarily shaped plasmonic channels and sharp bends," Phys. Rev. B 78, 035440 (2008).

[3] M. Kauranen, and A. V. Zayats, "Nonlinear plasmonics," Nat. Photon. 6, 737-748 (2012).

[4] C. Argyropoulos, P. Y. Chen, G. D'Aguano, N. Engheta, and A. Alù, "Boosting optical nonlinearities in epsilon-near-zero plasmonic channels," Phys. Rev. B 85, 045129 (2012). 